\title{
Effect of selenium on human prostate cell extracellular matrix
}

\author{
R. Hurst and S. J. Fairweather-Tait \\ School of Medicine, Health Policy and Practice, University of East Anglia, Norwich NR4 7TJ, Norfolk, UK
}

\begin{abstract}
A form of Se with anti-cancer benefit, Se-methylselenocysteine, found in Se-enriched alliums including onions and garlic has greater anticancer activity compared with other forms of $\mathrm{Se}^{(1)}$. Se-methylselenocysteine may alter the extracellular matrix through modulation of key proteins including members of the collagen family, and it has previously been shown that Se-methylselenocysteine can modulate the expression of several genes encoding different collagen subtypes ${ }^{(2)}$. A method has been developed for immunofluorescence imaging of collagen type I and VI in the extracellular matrix of cultured prostate cells. Prostate cells (LNCaP clone FGC) were maintained in Dulbecco's modified essential media with nutrient mix F12 (GIBCO ${ }^{\circledR}$; Invitrogen Ltd, Paisley, UK), glutamine plus $10 \%$ (v/v) serum at $37^{\circ} \mathrm{C}$ and $5 \% \mathrm{CO}_{2}$. The cells were grown on sterile coverslips for $3 \mathrm{~d}$ in the presence or absence of Se-methylselenocysteine and the expression of collagen subtypes was determined using immunofluorescence methods ${ }^{(3,4)}$ with a laser-scanning confocal microscope (Zeiss LSM450 META; Carl Zeiss MicroImaging GmbH, Jena, Germany). The different collagen subtypes were labelled with specific primary antibodies to collagen type I and type VI (Chemicon ${ }^{\circledR}$; Millipore, Billerica, MA, USA) and subsequently with secondary antibodies labelled with AlexaFluor 488 and 568 fluorophore dyes (Invitrogen Ltd) to study the expression of the two different types of collagen and the effect of Se on expression (Figure). By imaging collagen distribution in the extracellular matrix of cultured prostate cells following treatment with Se-methylselenocysteine or control an increase was observed in collagen type VI and a decrease in collagen type I in response to Se-methylselenocysteine relative to the control.
\end{abstract}
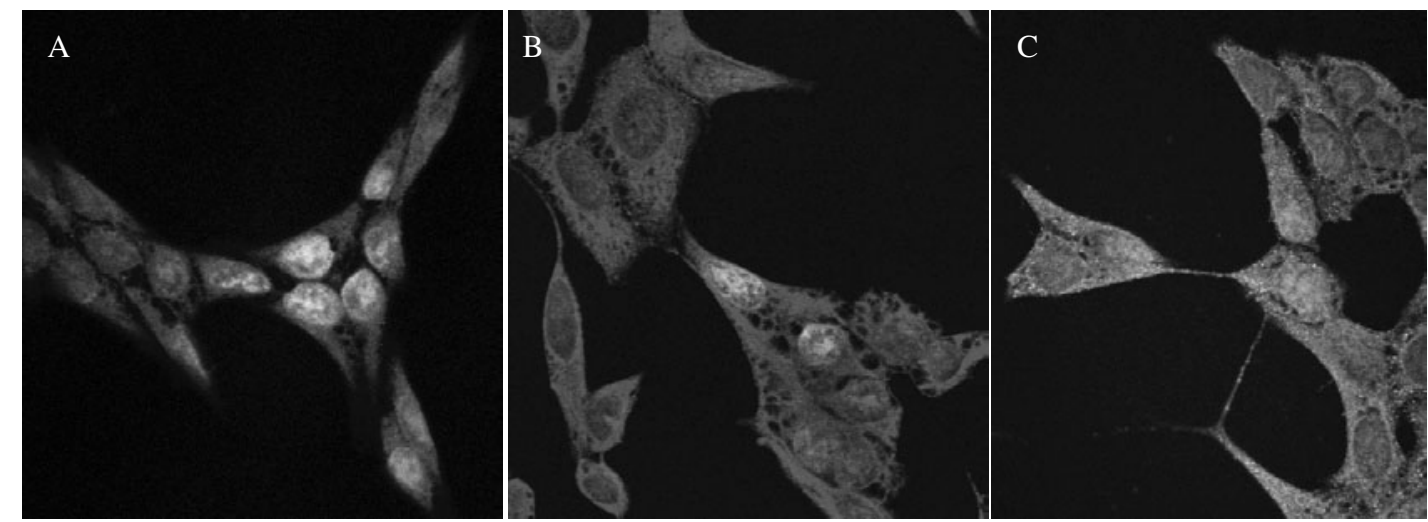

Figure. Example images to show effect of Se-methylselenocysteine (MSC) dose on collagen type VI and type 1 protein expression in prostate cells using immunofluoresence techniques. Collagen type VI is labelled with A568 (red) and collagen type 1 is labelled with A488 (green), nuclei labelled with DAPI (blue). Collagen type VI expression in prostate cell control (A), prostate cells + $500 \mathrm{~nm}-\mathrm{MSC}$ (B) and example of dual immunostaining for collagen type I and collagen type VI expression (C).

These data show that a dietary form of Se can alter the collagen profiles in the extracellular matrix of prostate cells. Since alterations in levels of various types of collagen play a key role in the reactive stroma effect in prostate cancer, where collagen remodelling and increased production of certain types of collagen, including collagen type $\mathrm{I}^{(5)}$, result in increased cancer cell proliferation and progression, with increased risk of metastases, the anti-cancer effects of Se may in part be mediated through the reported effects on the extracellular matrix proteins.

We thank the University of East Anglia for funding.

1. Ip C, Hayes C, Budnick RM et al. (1991) Cancer Res 51, 595-600.

2. Hurst R, Elliott RM, Goldson AJ et al. (2008) Cancer Lett 269, 117-126.

3. Ko MHK \& Kay EDP (2002) Mol Vis 8, 1-9.

4. Ko MHK \& Kay EDP (2005) Invest Opthal Mol Vis Sci 46, 4495-4503.

5. Tuxhorn JA, Ayala GE, Smith MJ et al. (2002) Clin Cancer Res 8, 2912-2923. 